\title{
The change of hospital internal medicine: a study on patients admitted in internal medicine wards of 8 hospitals of the Lazio area, Italy
}

\author{
Umberto Recine, ${ }^{1}$ Emilio Scotti, ${ }^{2}$ Vincenzo Bruzzese, ${ }^{3}$ Francesco D'Amore, ${ }^{2}$ Dario Manfellotto, ${ }^{4}$ Ilaria Simonelli, ${ }^{5,6}$ \\ Ruggero Pastorelli ${ }^{7}$ on behalf of GIMI - FADOI Lazio
}

${ }^{1}$ Department of Internal Medicine, S. Spirito in Saxia Hospital, Rome; ${ }^{2}$ FADOI Lazio Honorary President, Rome; ${ }^{3}$ Department of Internal Medicine, Nuovo Regina Margherita Hospital, Rome; ${ }^{4}$ Department of Internal Medicine, Fatebenefratelli Hospital, Rome; ${ }^{5}$ Department of Neuroimaging, IRCCS San Raffaele Pisana, Rome; ${ }^{6}$ Medical Statistics \& Information Technology, AFaRFatebenefratelli Hospital San Giovanni Calibita, Rome; ${ }^{7}$ Internal Medicine, L.P. Delfino Hospital, Colleferro (RM), Italy

\begin{abstract}
The hospital internal medicine (IM) needs to adapt to the socio-demographic changes occurred during the last thirty years: patients currently show an increased overall complexity owing to the increase in the average age of the patients admitted, to more several and severe comorbidities, and a higher concentration in our wards of most severe cases. Our departments have to change in order to pursue a more efficient organization and to offer care to users modulated according to their needs and conditions. The Intensity of Care in Internal Medicine Group of the Federation of Associations of Hospital Doctors on Internal Medicine - Lazio carried out a population-based study in eight Internal Medicine wards of the region with the aim of characterizing the patients there admitted, using the modified early warning score (mEWS), monitoring and evaluating the outcomes of hospitalization. This has allowed us to determine the cut-off of the score indicating a greater statistical probability of a fatal outcome corresponding to 3 , contrary to what originally reported by Subbe et al. in 2001. According to our results into the Departments of IM should be provided an area of high care, where monitor and stabilize the patients admitted with a mEWS score $\geq 3$, before transferring them to the wards of lower intensity of care. This organizational model of the high care of medical patients has the benefit of offer the technical and professional assistance appropriate to the level of clinical risk, with more intensive care to more critical stages of illness.
\end{abstract}

\section{Introduction}

Over the past 20 years, the welfare requirements of the population have undergone profound changes,

Correspondence: Umberto Recine, S. Spirito in Saxia Hospital, Lungotevere in Saxia 1, 00195 Rome, Italy.

Tel./Fax: +39.06.68352273.

E-mail: umberto.recine@asl-rme.it

Key words: Intensity of care driven assistance; internal medicine; early warning score.

Acknowledgments: the authors acknowledge Dr. Patrizio Pasqualetti, Medical Statistics \& Information Technology, AFaR-Fatebenefratelli Hospital S. Giovanni Calibita, Rome, for data statistical analysis.

See online Appendix for Members of Gruppo Intensità di Cura in Medicina Interna (GIMI) - FADOI Lazio.

Received for publication: 19 June 2014.

Revision received: 3 December 2014.

Accepted for publication: 30 December 2014.

This work is licensed under a Creative Commons Attribution NonCommercial 3.0 License (CC BY-NC 3.0).

(C) Copyright U. Recine et al., 2015

Licensee PAGEPress, Italy

Italian Journal of Medicine 2015; 9:252-259

doi:10.4081/itjm.2015.523 both in type and intensity, most of our hospitals struggled to adapt to this need, even having to combining the changes with the sustainability of the system. ${ }^{1}$

In particular, the medical area, rather than the internal medicine (IM) in itself, has to face new requests characterized by the increased complexity and heterogeneity of the cases, several technological innovations, patients' and their families empowerment, all this with the social necessity of maintaining the sustainability of the system..$^{2-4}$ The IM must therefore change its traditional production processes in the flows of patients, in order to maximize effectiveness and efficiency. ${ }^{4,5}$

In the literature a lot of studies demonstrate the benefits of redesigning the hospital organization as models of intensity of care, by using, at the time of admittance to the ward, some tools as prognostic scores, in order to identify the clinical risk of the patient, for which his allocation in a specific area of higher care may be appropriate. ${ }^{6-8}$

The Intensity of Care in Internal Medicine Group of the Federation of Associations of Hospital Doctors on Internal Medicine - Lazio (GIMI-FADOI Lazio) wanted to assess the complexity of treated cases, by promoting an observational study on the population admitted to $8 \mathrm{IM}$ units of the Lazio region in order to achieve a snapshot about the assisted users, as a basis for the analysis of needs. ${ }^{9}$ One secondary target of the 
study was to check the applicability and the impact of accuracy in our departments of a validated instrument such as the modified early warning score (mEWS) of Subbe, ${ }^{10}$ which provides 5 parameters: systolic and diastolic blood pressure values, body temperature, heart rate, respiratory rate (RR) and level of consciousness.

\section{Materials and Methods}

To understand the IM actual patient complexity, eight IM units from hospitals primarily in Rome cooperated to the present prospective study consisting in evaluating a cohort of consecutive patients admitted to medical wards in 2 periods of 3 months each, September, October and November 2011 and March, April and May 2012. A triage with the mEWS score was performed by the internist on duty at the IM admission on 1103 patients no matter if or how they were on treatment for their conditions. In addition to five parameters provided by the mEWS score, we detected three more clinical items: age, comorbidity and oxygen saturation $\left(\mathrm{SO}_{2}\right)$, that we wanted to test for sensitivity and specificity according to the outcome and the possible added value to the basic mEWS. The outcome has been classified in two categories: favorable, in case of discharge at home or to chronic, palliative or rehabilitative institutions for recovery or in the best achievable conditions, or unfavorable, meaning death or Intensive Care Unit (ICU) transfer.

\section{Statistical analysis}

In order to establish the relevance of the mEWS to predict patient outcome in IM, of adjunctive clinical variables and of the presence of the Emergency Department (ED) in hospital, we conducted a multivariable logistic regression analysis. The cut-off of 5, proposed by Subbe for patients admitted to ED, has been applied to IM population, and compared to some other cut-off values, potentially more suitable for our series.

Again, through multivariate logistic regression, the role of additional items proposed by the research team was assessed.

The correlation between parameters was assessed using the Spearman correlation coefficient. A P-value $<0.05$ was considered significant. Statistical analyses were carried out with SPSS 16.0 statistical software (IBM Corp., Armonk, NY, USA).

\section{Results}

Out of the total 1103 patients, 940 were admitted to hospitals with ED and 163 in structures without ED (Table 1). The median hospital stay was 7 days (1-84). Of the 1103 received folders, $303(27.4 \%)$ were incomplete as far as the missed outcome record is con- cerned and therefore used only for the entry data. Of the 800 patients with full reports, 718 were hospitalized in IM departments of hospitals with ED and the remaining 82 in hospitals without ED. Mortality was $12 \%$ in the first group, $4.9 \%$ in the second, confirming a greater severity of patients admitted in the structures equipped with $\mathrm{ED}$ [relative risk $=2.7,95 \%$ confidence interval $(\mathrm{CI})=1.01,7: 09, \mathrm{P}=0.034]$. A similar result was observed considering the incidence of an unfavorable outcome, $13.1 \%$ in the first group vs. again $4.9 \%$ in the latter. The admission mEWS ranged between 0 to 14 , with a median of 1 (Figure 1). 36.6\% had a score $0 ; 20.7 \% \geq 3$. The presence or absence of ED also affects the distribution of the mEWS (MannWhitney, $\mathrm{P}<0.001$ ), with higher scores in wards of the hospitals with ED, where $22.6 \%$ of patients had an admission mEWS $\geq 3$ vs $9.9 \%$ of those without ED. Through a logistic regression model with an unfavorable outcome as the event of interest and mEWS score and presence/absence of ED as potential predictors, we found that the effect on outcomes for the presence of ED is absorbed by the mEWS score.

The receiver operator characteristic curve (ROC) (Figure 2) has allowed evaluating the sensitivity and specificity at the change of the cut-off values. The cut-

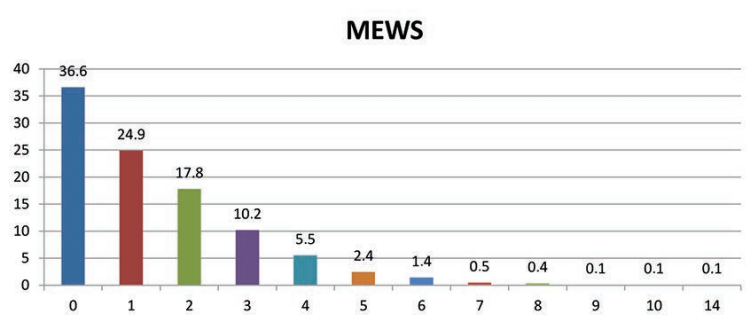

Figure 1. Modified early warning score (mEWS) score on 1103 patients enrolled.

Table 1. Involved Institutes.

\begin{tabular}{lcc}
\hline Institutes & $\begin{array}{c}\text { Patients (\%) } \\
\text { included in the study }\end{array}$ & ED \\
\hline S. Spirito Hospital & 43.1 & Yes \\
\hline Hospital of Colleferro & 14.4 & Yes \\
\hline S. Camillo Hospital & 9.4 & Yes \\
\hline Sandro Pertini Hospital & 9.3 & Yes \\
\hline Vannini-Figlie di S. Camillo Hospital & 9.3 & Yes \\
\hline S. G. Calibita-Fatebenefratelli Hospital & 1.6 & Yes \\
\hline L. Di Liegro Polyclinic & 7.3 & No \\
\hline Civile Hospital of Marino & 5.4 & No \\
\hline ED, Emergency Department. & &
\end{tabular}


off of 5 proposed in the article by Subbe et al. would imply a high specificity $(96.7 \%$, for which only $3.7 \%$ of patients with a favorable outcome would have a score 5) but a low sensitivity (21\%, for which $79 \%$ of patients with an unfavorable outcome would have a score less than 5). The cut-off which was more useful to the case mix of units of IM appeared to be 3 (sensitivity $=72 \%$, specificity $=62 \%$ ).

In addition to the revaluation of the cut-off for a better adaptation to the IM clinical context we also carried out an evaluation of the usefulness of each individual items of the mEWS and the benefit of the addition of three other items, age, comorbidity and $\mathrm{SO}_{2}$.

With regard to the assessment of the mEWS, we observed (Table 2) that in univariable regressions all five items have shown significant results, while in the multi-variable model, the body temperature and heart rate items have not shown any additional predictive ability, compared to the cluster of blood pressure, respiration and state of consciousness. In other words, in our IM series we could also reduce the mEWS criteria in 3 from the 5 original items. However, for a comparison with the literature, we considered the original mEWS score to check the possible contribution of some additional items. As shown in Table 3, age (starting from 85 years old), oxygen saturation (for values between 86 and 92) and only marginally the number of comorbidities are associated in univariable analysis with unfavorable events. However, the multivariable analysis showed that the contribution only of age is statistically significant in terms of predicting unfavorable outcomes, in addition to the mEWS score. Looking at the ROC curves does not emerge any clear gain in terms of predictive accuracy with the addition of age and $\mathrm{SO}_{2}$ to the original $\mathrm{mEWS}$ score.
The graphical representation is supported by the lack of a significant effect in the comparison of the ROC curves for paired data $(\mathrm{P}>0.05$ for each of the comparisons). Table 4 shows sensitivity and specificity values for the four cut-off suggested scores.

In a previous survey carried out in a single center (L'assistenza per intensità di cura: progetto di riorganizzazione dell'area medica per intensità di cura nell'Ospedale S. Spirito in Saxia - Roma, personal communication, 2012), which used the same mEWS admission score for the definition of the clinical risk of IM in patients admitted from ED, Observation Unit, Cardiology, Surgery or Intensive Care Unit, out of 178 consecutive patients over a period of four months, the score 2 and 3 entailed respectively a probability of an adverse event of 23.5 and $71.4 \%$, while the risk of the patients with a score of two and three together resulted $37.4 \%$.

By analyzing the results of the logistic model we have observed some interesting information: even if all parameters, except for heart rate 101-110, body temperature $>38.5^{\circ} \mathrm{C}$, age $<80$ years and comorbidities (Table 5) were significant indicators of risk of adverse events (OR), systolic arterial pressure (SAP) $<70 \mathrm{mmHg}$ (31.6) and alert, voice, pain, unresponsive (AVPU) scale have the most impact compared to others. In the AVPU assessment, however, the reactivity to pain or lack of any response have similar ORs (11.4 and 11.7).

The three additional criteria tested did not appear to improve sensitivity and specificity of the mEWS, which seems to work just as well if limited to only the three parameters SAP, heart rate (HR) and AVPU.

The $\mathrm{SO}_{2}$ is certainly a matter of importance, even if the risk does not appear substantially different between $86-92$ and $70-85 \% \mathrm{SO}_{2}$ values. The same can be said about RR in which the risk between 21-29 and
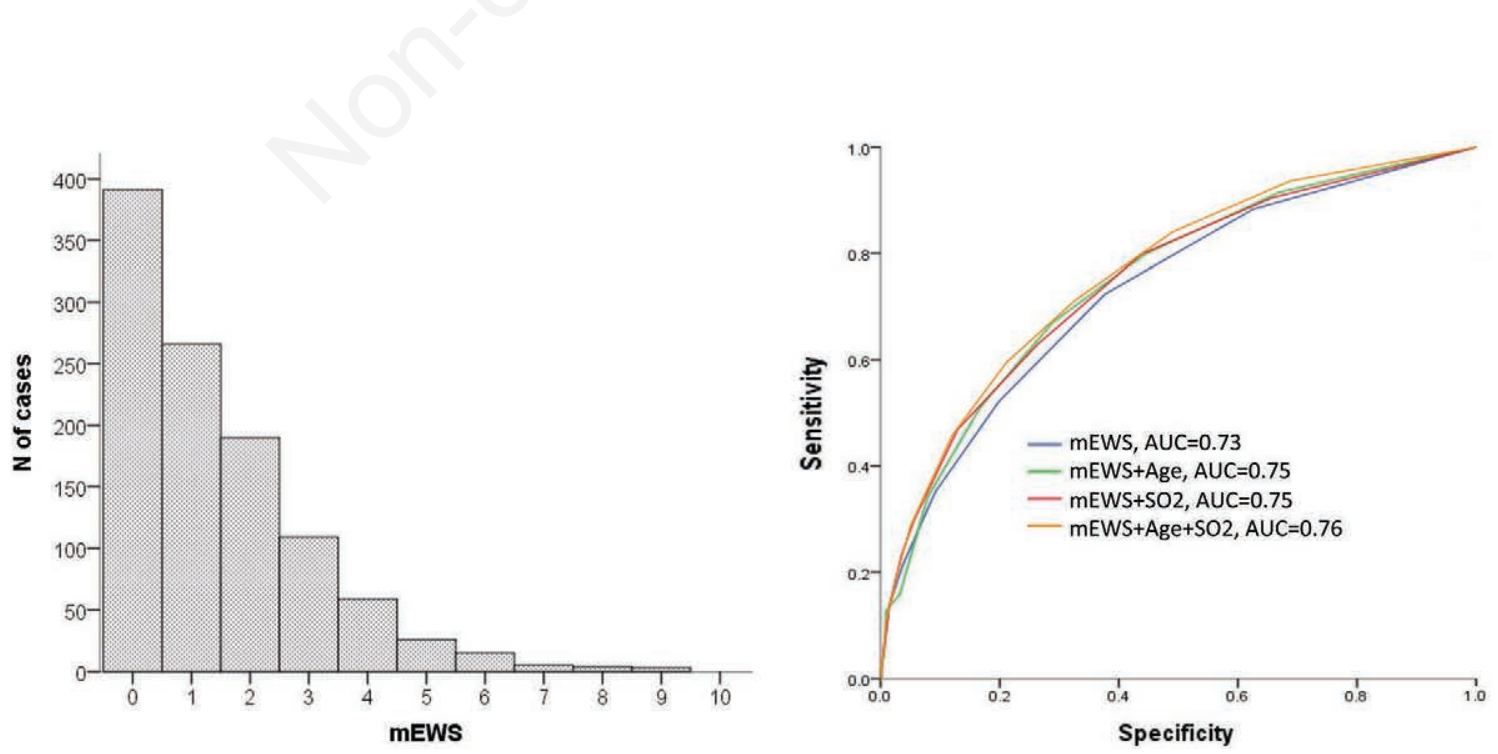

Figure 2. Receiver operator characteristic curve (ROC) for modified early warning score (mEWS) and mEWS with age score, with age and comorbidity score and with age, comorbidity and oxygen saturation $\left(\mathrm{SO}_{2}\right)$ score. AUC, area under the curve. 
over 29 breaths/min is similar. The body temperature is confusing, since it seems to have more weight for values of 37.5 to $38.5^{\circ} \mathrm{C}$ compared to $>38.5^{\circ} \mathrm{C}$. Moreover, the hyperpyrexia, resulting in the variation of other parameters included in the mEWS as RR and HR can often determine an initial score of 3 or 4 , which does not correspond to the same risk of a coma or severe hypotension, as noted by Bulut et al. ${ }^{11}$ In addition, the factor related to blood pressure is difficult to assess, since it is hard to imagine that SAP 161-200 or diastolic arterial pressure (DAP) 96-110 have the same risk compared to SAP 71-80, both getting score

Table 2. Effects of single modified early warning score item on incidence of unfavorable outcome.

\begin{tabular}{|c|c|c|c|c|}
\hline \multirow[b]{2}{*}{ mEWS items } & \multicolumn{2}{|c|}{ Univariable } & \multicolumn{2}{|c|}{ Multivariable } \\
\hline & $\mathbf{O R}$ & P-value & OR & P-value \\
\hline \multicolumn{5}{|l|}{ Arterial pressure mmHg } \\
\hline SAP $101-160$ and DAP $<95$ & 1 & $<0.001$ & 1 & $<0.001$ \\
\hline SAP $81-100$ or SAP $161-200$ and DAP $96-110$ & $2.9(1.8-4.6)$ & $<0.001$ & $2.1(1.2-3.6)$ & 0.008 \\
\hline $\mathrm{SAP}<80$ or $\mathrm{SAP}>200$ or $\mathrm{DAP}>110$ & $7.9(3.1-19.6)$ & $<0.001$ & $6.1(2.0-18.4)$ & 0.001 \\
\hline \multicolumn{5}{|l|}{ Heart rate bpm } \\
\hline $60-100$ & 1 & 0.036 & - & 0.304 \\
\hline $41-59$ or $101-110$ & $1.2(0.6-2.3)$ & 0.597 & - & - \\
\hline $111-129$ & $2.3(0.9-5.9)$ & 0.082 & - & - \\
\hline$\geq 130$ or $<40$ & $3.4(1.3-9.3)$ & 0.014 & - & - \\
\hline \multicolumn{5}{|l|}{ Respiratory rate/min } \\
\hline $9-14$ & 1 & $<0.001$ & 1 & 0.007 \\
\hline $15-20$ & $2.6(1.5-4.5)$ & $<0.001$ & $1.8(1.0-3.4)$ & 0.048 \\
\hline $21-29$ & $3.4(1.8-6.2)$ & $<0.001$ & $2.7(1.4-5.4)$ & 0.004 \\
\hline$>29$ & $3.4(1.5-7.4)$ & 0.003 & $3.4(1.4-8.2)$ & 0.008 \\
\hline \multicolumn{5}{|l|}{ Body temperature $\left({ }^{\circ} \mathrm{C}\right)$} \\
\hline $35-37.5$ & 1 & 0.066 & - & 0.239 \\
\hline $37.5-38.5$ & $2.3(1.1-4.7)$ & 0.023 & - & - \\
\hline$>38.5$ & $1.5(0.4-5.4)$ & 0.503 & - & - \\
\hline \multicolumn{5}{|l|}{ AVPU } \\
\hline Alert & 1 & $<0.001$ & 1 & $<0.001$ \\
\hline Reactive to voice & $3.7(1.9-7.0)$ & $<0.001$ & $3.6(1.8-7.2)$ & $<0.001$ \\
\hline Reactive to pain & $11.4(4.4-29.2)$ & $<0.001$ & $10.0(3.4-29.7)$ & $<0.001$ \\
\hline Unreactive & $11.7(4.1-33.5)$ & $<0.001$ & $6.0(1.9-18.7)$ & 0.002 \\
\hline
\end{tabular}

OR, odds ratio; mEWS, modified early warning score; SAP, systolic arterial blood pressure; DAP, diastolic arterial pressure; AVPU, alert, voice, pain, unresponsive scale.

Table 3. Modified early warning score and single added item value on unfavorable outcome.

\begin{tabular}{lcccc}
\hline & \multicolumn{2}{c}{ Univariable } & \multicolumn{2}{c}{ Multivariable } \\
\hline Additional items & OR & P-value & OR & P-value \\
\hline mEWS score & $1.59(1.39-1.83)$ & $<0.001$ & $1.57(1.37-1.81)$ & $<0.001$ \\
\hline Age & & & & \\
$<75$ & 1 & $<0.001$ & 1 & 0.01 \\
$75-84$ & $1.3(0.6-2.6)$ & 0.546 & $0.9(0.4-2.3)$ & 0.839 \\
$85-89$ & $2.0(1.1-3.5)$ & 0.021 & $2.0(0.9-3.9)$ & 0.059 \\
$>89$ & $3.6(1.9-7.0)$ & $<0.001$ & $3.3(1.5-7.5)$ & 0.004 \\
\hline SO & & & & - \\
$>92$ & 1 & $<0.001$ & - & - \\
$86-92$ & $3.2(1.9-5.2)$ & $<0.001$ & - & - \\
$\leq 85$ & $4.1(2.0-8.4)$ & $<0.001$ & - & - \\
\hline Comorbidities & & & & - \\
$0-2$ & 1 & 0.12 & - & - \\
$3-4$ & $1.6(0.9-2.9)$ & 0.114 & - & - \\
$>6$ & $2.2(1.1-4.7)$ & 0.029 & - & - \\
\hline
\end{tabular}

OR, odds ratio; mEWS, modified early warning score. 
2 in mEWS. Anyway, fever and arterial pressure show a certainly lighter weight than parameters as AVPU or $\mathrm{HR}<40$, although in the mEWS they have an equivalent value (Table 4). Surprisingly, the comorbidity did not appear related with adverse prognosis, ${ }^{12}$ whereas age higher than 85 was significantly associated with an adverse event. ${ }^{13}$

\section{Discussion}

The change in complexity of the patients admitted in IM wards is due to multiple causes: i) the aging population with multiple comorbidities and polypharmacy phenomenon; ii) the increasing migration from non-EU countries and mass tourism for extraordinary events; iii) more chronic diseases overlapped to functional dependence, psycho-cognitive and socio-economic dysfunctions; iv) conservative shift to medical areas of some in the past surgical diseases; $v$ ) the increase of diagnostic and therapeutic technological development; vi) the exasperated search of efficiency in health care system, with contraction of resources and hospital beds; vii) the influence on the diagnostic and therapeutic choices of the economic context; and viii) the increasing of the defensive medicine.
On the other hand, in IM departments is hospitalized an highly heterogeneous population of patients, with different health care needs: critical patients or with an unstable state, with patients with multiple chronic diseases and/or socio-environmental problems, ${ }^{14,15}$ in a context not modulated for their different requirements. Our units do not provide adequate resources nor technologies addressed to patients with different clinical risks. This is currently one of the main obstacles to the provision of a qualitatively and quantitatively appropriate care or adjusted according to the true patient needs. On the other hand, we have not yet a strong enough evidence of a sure positive impact of an intensity of care organization on the IM patient quality assistance and outcome, but in some limited studies. . $^{16,17}$

In recent years, the internist central role has been reaffirmed, mainly in hospitals of small to medium size, where IM collects patients from all medical subspecialties. In these situations the internist is de facto the director of the diagnosis and treatment, using, adapting them to each clinical picture, subspecialist's indications, when needed. Often the patients in IM are elderly or very elderly, but, according to this, only internists are able to combine the judgment on life expectancy with its quality with sustainable diagnostic and therapeutic pathways. ${ }^{18,19}$ In our population the

Table 4. Sensitivity and specificity of the optimal cut-off (Youden index) for the original modified early warning score and integrated with additional items.

\begin{tabular}{|c|c|c|c|}
\hline Score & Optimal cut off & Sensitivity \% & Specificity \% \\
\hline mEWS & $\geq 3$ & 72 & 62 \\
\hline mEWS+age & $\geq 4$ & 67 & 71 \\
\hline $\mathrm{mEWS}+\mathrm{SO}_{2}$ & $\geq 3$ & 63 & 74 \\
\hline $\mathrm{mEWS}+$ age $+\mathrm{SO}_{2}$ & $\geq 4$ & 71 & 67 \\
\hline
\end{tabular}

mEWS, modified early warning score; $\mathrm{SO}_{2}$, oxygen saturation score.

Table 5. Analysis of some parameters and relative risk.

\begin{tabular}{llc}
\hline Parameters & Relative risk \\
\hline $\mathrm{SAP}<80 v s \geq 80 \mathrm{mmHg}$ & 5.74 & 31.5 \\
\hline $\mathrm{SAP}<70 v s \geq 80 \mathrm{mmHg}$ & 3.39 \\
\hline $\mathrm{HR}>21$ vs $9-14 / \mathrm{bpm}$ & $2.29 /-1.9$ \\
\hline $\mathrm{T}\left({ }^{\circ} \mathrm{C}\right)>37.5$ and $>38.5$ s $35-37.5$ & 3.66 \\
\hline Reactive to voice coma $v s$ alert & $13.5-13.9$ \\
\hline Reactive to pain coma or not reactive $v s$ alert & $2.8 /-5.2$ \\
\hline Age $81-89$ and $\geq 90 v s<75$ years & $3.15 /-3.49$ \\
\hline SO $26-92$ and $70-85 v s>92 \%$ & 2.3 \\
\hline Comorbidities $\geq 5 v s \leq 2$
\end{tabular}

SAP, systolic arterial blood pressure; $\mathrm{HR}$, heart rate; $\mathrm{T}$, temperature; $\mathrm{SO}_{2}$, oxygen saturation score. 
presence of ED distinguishes substantially the complexity of cases treated in IM: patients are most frequently critical, with increased risk of death or adverse event, compared to the structures without ED, where requests of low intensity care are prevalent. Although the majority of people admitted in IM have a mEWS risk score 0 to 1 , there is a plot around $15-20 \%$ of patients who require close clinical and instrumental monitoring, in order to promptly detect and treat any negative development of the clinical picture. This population is not appropriate for the ICU because it does not require ventilation neither other invasive procedures, but may need more complex and higher technology supports for preventing or treating the failure of one or more organs.

For these patients, we should provide an area in the IM wards with trained professionals and instrumental equipment, in order to deliver a high intensive care, the high care area Internal Medicine. Its task is to stabilize the patient before transferring him to the standard area. We should strive to make more flexible our IM units, by means of structural, educational and technical changes that allow us to graduate the intensity of the provided care. ${ }^{20}$ This would result in net savings of days of hospitalization in the ICU, where they are often improperly admitted, with economic advantages for the management of the hospital. ${ }^{21,22}$ In addition to specific organizational pathways, that have to be built together with ED units for diseases at higher risk of acute organ failure as indicated above, it is therefore essential, at the admission in the ward and throughout the course of hospital stay, a widely accepted, easily reproducible risk stratification system (triage) of the patients, to provide the better adequate care related to their actual needs. ${ }^{23-26}$ For this purpose the IM adapted mEWS proved to be a simple and effective tool. ${ }^{27,28}$

We share the opinion by Chesi and Nardi, ${ }^{24}$ for which the stratification of patients for different clinical risk in IM should not be based only on vital parameters, since there are medical conditions, although with lower scores at admission, that are at higher risk of organ failure or sudden worsening, requiring, regardless of the score at admission, a close clinical monitoring. Some examples of these conditions include shock of various etiology, cardiac rhythm or conduction disorders that require monitoring, systemic inflammatory response syndrome or sepsis and others listed in Table $6 .{ }^{29}$ Listed conditions make a provisional list to be modified according to the real life working evidence of necessary changes.

\section{Conclusions}

On the basis of our findings, in order to improve the sensitivity and specificity of the mEWS we propose to test a 6-parameter clinical risk score for Internal Medicine, derived from mEWS excluding body temperature, as already suggested in the rapid emergency medicine score, ${ }^{28}$ and the high values of SAP and DAP, adding age and $\mathrm{SO}_{2}$, joining the unresponsive (U) and responsive to pain $(\mathrm{P})$ coma and attributing a different weight to the $\mathrm{HR}<40 / \mathrm{min}$ and $>130 / \mathrm{min}$.

Every item has 3 values (Table 7) with a cut-off final score distinguishing between standard and high clinical risk for IM patients to be determined. It could be possibly 2 or more for intensive care candidate. Moreover, besides the score, that everybody consider

Table 6. Clinical conditions needing a higher care.

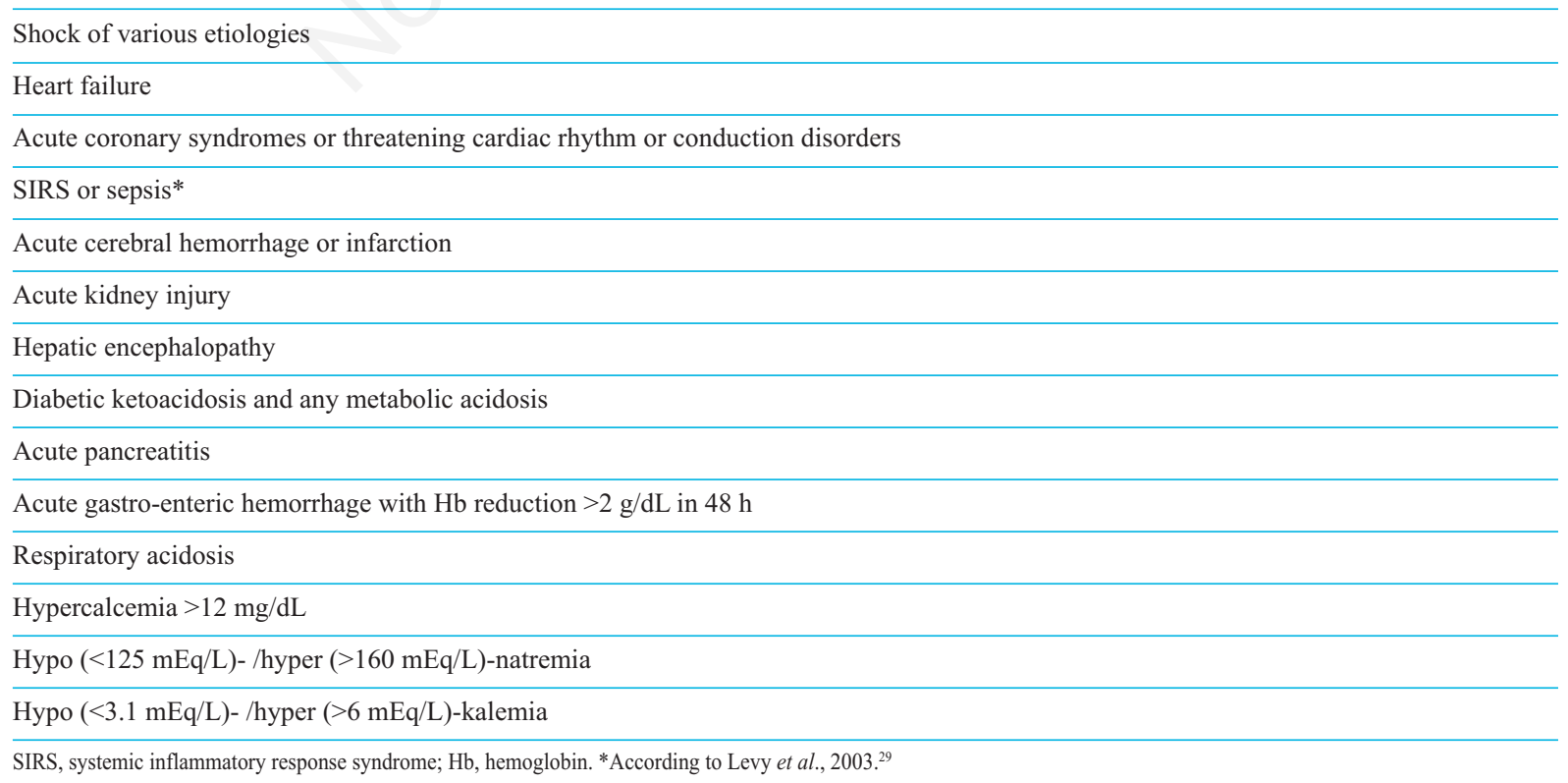


Table 7. Proposed clinical risk score for internal medicine patients.

\begin{tabular}{lccc}
\hline & $\mathbf{0}$ & $\mathbf{1}$ & $\mathbf{2}$ \\
\hline $\mathrm{SAP}$ & $>85$ & $80-85$ & $<80$ \\
\hline $\mathrm{HR}$ & $<130$ & $>130$ & $<40$ \\
\hline $\mathrm{RR}$ & $<24$ & $>24$ & - \\
\hline $\mathrm{Age}$ (years) & $<80$ & $80-89$ & $\geq 90$ \\
\hline $\mathrm{AVPU}$ & Alert or arousable & Reactive to voice & Reactive to pain or not reactive \\
\hline $\mathrm{SO}_{2}(\%)$ & $\geq 90$ & $85-89$ & $<85$ \\
\hline Date & \multicolumn{2}{c}{ Total score } & \\
\hline
\end{tabular}

SAP, systolic arterial pressure; $\mathrm{HR}$, heart rate; $\mathrm{RR}$, respiratory rate; $\mathrm{AVPU}$, alert, voice, pain, unresponsive scale; $\mathrm{SO}_{2}$, oxygen saturation score.

not to be exhaustive for defining the patient clinical status, ${ }^{15}$ we suggest to add to it the presence or suspect of one of the listed clinical conditions that, when present, by itself make necessary a medical higher care area, until clinical stabilization. In other words, any of the listed conditions overwhelm the numeric score and should be considered in the proposed evaluation form. We are concerned about the difficulty to propose an evidence based and universally valid list of critical conditions fitting to various Internal Medicine units operating in different contexts with different facilities. This is why any medicine unit should cooperate to build a pathology list to use aside the score, adding or removing conditions as far as its own needs are concerned, with the common purpose to reach the most locally useful tool for each operative unit. In summary, the proposed list has a provisional value and it needs to be proved on field and changed according to as many IM teams experience and organizational necessities as possible.

The proposal of our study group (GIMI-FADOI Lazio) is to test the modified score together with the proposed list of pathological condition in prospective comparison to mEWS. The test should be applied both at the admission in IM department and in the course of hospitalization, at any change in clinical condition. The assessment should be carried out again at discharge, indicating the outcome, according to the terms favorable or unfavorable applied in this study, in an appropriate number of IM units of Italian hospitals with ED. This could provide a shared tool for homogeneous assessment of our patient population, in order to make comparable multicenter case studies exploring the complexity of our patients.

\section{References}

1. Murray CJL, Lopez AD. Measuring the global burden of disease. NEJM 2013;369:448-57.

2. Lega F, Villa S, Barbieri M. Restructuring patient flow logistics around patient care needs: implications and practicalities from three critical cases. Health Care Manage Sci 2009;12:155-65.

3. Lega F, De Pietro C. Converging patters in hospital organizzation: beyond the professional bureaucracy. Health Policy 2005;74:261-81.

4. Steinman MA, Auerbach D. Managing chronic disease in hospitalized patients. JAMA 2013;173:678-9.

5. Di Napoli M. Ospedale per intensità di cura in Medicina Interna. Il Policlinico Sez Prat 2011;118:79-85.

6. Alessandri M, Bartolomei C, Bernardini M, et al. Medicina Interna e nuova organizzazione ospedaliera: la proposta della FADOI Toscana. Ital J Med 2007;1:65-9.

7. D'Amore F. Proposta d'organizzazione delle U.O. di Medicina Interna secondo il sistema "Hub \& Spoke". Il Policlinico Sez Prat 2010;117:405-10.

8. Helay J, McKee M. Hospital in a changing Europe. Buckingham: Open University Press; 2002.

9. Puleo A, Di Rosa S. Tipologia del paziente critico in Medicina Interna. Giorn Ital Med Int 2002;1:33-7.

10. Subbe CP, Kruger M, Rutherford P, et al. Validation of a modified early warning score in medical admission. Q J Med 2001;94:521-6.

11. Bulut M, Cebicci H, Sigirli D, et al. The comparison of modified early warning score with rapid emergency medicine score: a prospective multicentric observational cohort study on medical and surgical patients presentino to emergency department. Emerg Med J 2014;31:476-81.

12. Scotti E, Pietrantonio F. The hospital Internal Medicine specialist today: a literature review and strength, weaknesses, opportunity, threats (SWOT) analysis to develop a working proposal. Ital J Med 2013;7:278-86.

13. Nobili A, Marengoni A, Tettamanzi M, et al. Association between clusters of diseases and polypharmacy in hospitalized elderly: results from the REPOSI study. Eur J Intern Med 2011;22:597-602.

14. Nardi R, Berti F, Greco A, et al. Complexity in hospital internal medicine departments: what are we talking about? Ital J Med 2013;7:142-55.

15. Chiesi G, Boni F. Ospedali e modelli organizzativi per intensità di cure: il punto di vista dell'internista. Ital J Med 2012;6:63-71.

16. Nguyen Y, Wunsch H, Angus DC. Critical care: the impact of organization and management on outcomes. Curr Opin Crit Care 2010;16:487-92.

17. Lacson E Jr, Maddux FW. Intensity of care and better 
outcomes among hemodialysis patients: a role for the Medical director. Sem Dialysis 2012;25:299-302.

18. Smith AK, Lo B, Aronson L. Elder self-neglect - How can a physician help? NEJM 2013;369:2476-9.

19. Villa S, Barbieri M, Lega F. Restructuring patient flow logistics around patient care needs: implications and practicalities from three critical cases. H Care Manag Sci 2009,12;155-65.

20. Orlandi W, Duca E, Pioppo M. L'ospedale per aree di intensità di cura omogenee e di assistenza multi specialistica: l'esperienza dell'Azienda USL n.3 Umbria. Organ Sanit 2006;4;35-40.

21. Nardi R, Arienti V, Nozzoli C, et al. Organizzazione dell'ospedale per intensità di cura: gli errori da evitare. Ital J Med 2012;6:1-13.

22. Villa S, Barbieri M, Lega F. Aspetti concettuali ed operativi della logistica nelle aziende sanitarie. Il caso del Presidio Ospedaliero di Forlì. MeCoSan 2007;61:127-58.

23. Nardi R, Scanelli G, Borioni D, et al. The assessment of complexity in internal medicine patients. The FADOI Micomplex Study. Eur J Intern Med 2007;18:283-7.

24. Chiesi G, Nardi R. Severity stratification of patients hos- pitalized on the internal medicine ward: work in progress. Ital J Med 2013;7:231-3.

25. Groarke JD, Gallagher J, Stack J, et al. Use of an admission early warning score to predict patient morbidity and mortality and treatment success. Emerg Med J 2008; 12:803-6.

26. Kellett J, Kim A. Validation of an abbreviated Vitalpac early warning score (ViEWS) in 75,419 consecutive admissions to a Canadian Regional Hospital. Resuscitation 2012;83:297-302.

27. Cei M, Bartolomei C, Mumoli N. In-hospital mortality and morbidity of elderly medical patients can be predicted at admission by the modified early warning score: a prospective study. Int J Clin Pract 2009;4:591-5.

28. Heitz CR, Gaillard JP, Blumstein H, et al. Performance of the maximum modified EWS to predict the need for higher care utilization among admitted emergency department patients. J Hosp Med 2010;5:e46-52.

29. Levy MM, Fink MP, Marshall JC, et al. 2001 SCCM/ ESICM/ACCP/ATS/SIS International sepsis definitions conference. Crit Care Med 2003;31:1250-6. 